\title{
Aphid-host plant interactions: does aphid honeydew exactly reflect the host plant amino acid composition?
}

\author{
Pascal D. Leroy $\cdot$ Bernard Wathelet $\cdot$ Ahmed Sabri • \\ Frédéric Francis · François J. Verheggen • \\ Quentin Capella $\cdot$ Philippe Thonart · Eric Haubruge
}

Received: 1 September 2010/ Accepted: 1 February 2011

(C) Springer Science+Business Media B.V. 2011

\begin{abstract}
Plants provide aphids with unbalanced and low concentrations of amino acids. Likely, intracellular symbionts improve the aphid nutrition by participating to the synthesis of essential amino acids. To compare the aphid amino acid uptakes from the host plant and the aphids amino acid excretion into the honeydew, host plant exudates (phloem + xylem) from infested and uninfested Vicia faba L. plants were compared to the honeydew produced by two aphid species (Acyrthosiphon pisum Harris and Megoura viciae Buckton) feeding on V. faba. Our results show that an aphid infestation modifies the amino acid composition of the infested broad bean plant since the global concentration of amino acids significantly increased in the host plant in response to aphid infestations. Specifically, the concentrations of the two amino acids glutamine and asparagine were strongly enhanced. The amino acid profiles from honeydews were similar for the two aphid species, but the concentrations found in the honeydews were generally lower than those measured in
\end{abstract}

Handling Editor: Gary Felton.

P. D. Leroy $(\bowtie) \cdot$ F. Francis · F. J. Verheggen · Q. Capella ·

E. Haubruge

Department of Functional and Evolutionary Entomology,

University of Liege, Gembloux Agro-Bio Tech,

Passage des Déportés 2, 5030 Gembloux, Belgium

e-mail: entomologie.gembloux@ulg.ac.be

B. Wathelet

Industrial Biological Chemistry Unity, University of Liege,

Gembloux Agro-Bio Tech, Passage des Déportés 2, 5030

Gembloux, Belgium

A. Sabri $\cdot$ P. Thonart

Walloon Center of Industrial Biology, University of Liege, B40,

4000 Sart-Tilman, Belgium the exudates of infested plants (aphids uptakes). This work also highlights that aphids take large amounts of amino acids from the host plant, especially glutamine and asparagine, which are converted into glutamic and aspartic acids but also into other essential amino acids. The amino acid profiles differed between the host plant exudates and the aphid excretion product. Finally, this study highlights that the pea aphid, a "specialist" for the $V$. faba host plant, induced more important modifications into the host plant amino acid composition than the "generalist" aphid M. viciae.

Keywords Amino acids · Phloem sap · Xylem sap · Aphid · Honeydew

\section{Introduction}

Aphids feed on the phloem and the xylem sap of plants (Spiller et al. 1990; Smith 1926; Saheed et al. 2007) and are the most common honeydew producing insects. This aphid excretory product consists in an aqueous mixture of different chemical compounds, of which the most important are sugars (90-95\% of the dry weight) and amino acids (Auclair 1963). Many studies have shown that the chemical composition of aphid honeydew varies with (1) the host plant species (Hendrix et al. 1992; Fischer and Shingleton 2001; Karley et al. 2002), (2) the nutritional state of the host plants (Mittler 1958; Douglas 1993), (3) the aphid species, developmental stage and age (Hendrix et al. 1992; Arakaki and Hattori 1998; Fisher et al. 2002; Sandström et al. 2000), (4) the rate and duration of aphid infestation (Faria et al. 2008), (5) the presence of ants (mutualism) (Yao and Akimoto 2001; 2002; Way 1963), (6) the presence of bacterial intracellular symbionts (Wilkinson et al. 
1997), (7) the parasitism state (Cloutier 1986) and the presence of secondary plant metabolites (Malcolm 1990). Plant-derived phloem sugars (67-89\% of the sugar content) such as glucose, fructose, sucrose or maltose and free amino-acids such as asparagine, glutamine, glutamate and serine (78\% of the amino acid content) seem to be universally present in honeydew (Hogervorst et al. 2007; Woodring et al. 2004). The sugar composition of honeydew reflects the composition of phloem sap but various other mono-, di-, and oligo-saccharides are also synthesized by the sap feeder (action of gut enzymes on plant derived sucrose) such as melezitose, erlose (fructomaltose), raffinose and trehalose (Wäckers 2000, 2005; Mittler 1958; Hendrix et al. 1992). The amino acid composition of the honeydew corresponds to the phloem sap content: asparagine and glutamine were reported as the two major amino acids in honeydew but also in the phloem sap of some plant species (Sasaki et al. 1990; Douglas 1993). Aphids exploit intracellular symbionts (endosymbionts) to incorporate nitrogenous wastes into essential amino acids or transform non essential amino acids in phloem sap into essential amino acids (Smith and Douglas 1987; Prosser and Douglas 1991). The main nitrogen sources of aphids are free amino acids found in very low and unbalanced concentrations in the phloem sap and it is well known that aphids, like other animals, cannot synthesize all the amino acids (Douglas 1993). Endosymbiotic bacteria from the genus Buchnera are therefore considered as indispensable, providing essential amino acids to their host (Febvay et al. 1999; Douglas 1998).

Only few studies take in consideration the xylem ingestion by aphids. Smith (1926) already showed that the aphid Mysus persicae Sulzer uses both xylem and phloem sap. Futhermore, aphids are known to drink periodically from the xylem (Tjallingii 1994) and Saheed et al. (2007) demonstrated that the aphid Diuraphis noxia Mordvilko exploited the xylem in barley plants. It has been suggested that during the day an aphid will accumulate a water deficit, as a result of losing water to its relatively concentrated gut contents (Cull and van Emden 1977). According to Spiller et al. (1990), xylem ingestion may be a common and efficient method of restoring and maintaining the water balance of the insect.

In this study, we simultaneously considered xylem and phloem sap and used the terms "host plant exudate" or "host plant content" to define these two aphid intakes.

The aim of this study was to compare the amino acid profiles from the host plant exudates to the profiles obtained with the honeydews of two aphid species (Acyrthosiphon pisum and Megoura viciae). Analyses were realised with exudates collected from uninfested and infested Vicia faba host plants in order to determine (1) how the amino acid exudate composition is modified when aphids feed on the host plant and (2) how these modifications affect the honeydew amino acid content.

\section{Materials and methods}

\section{Biological material}

In a climate-controlled room ( $16 \mathrm{~h}$ light photoperiod; $60-70 \% \mathrm{RH} ; 20 \pm 2{ }^{\circ} \mathrm{C}$ ), the host plants Vicia faba L. (var. Major) were grown in $9 \mathrm{~cm} \times 8 \mathrm{~cm}$ plastic pots containing a mixture of vermiculite and perlite (1/1) and were infested with 150 aphids Acyrthosiphon pisum Harris or Megoura viciae Buckton. Both aphid species were collected in field crops in 1990 and are reared for years at the University of Liege, Gembloux Agro-Bio tech (Department of Functional and Evolutionary Entomology). Aphids are weekly transferred on new $V$. faba host plants (grown in a mixture of vermiculite and perlite) and maintained in a climate-controlled room (16 h light photoperiod; $60-70 \%$ $\mathrm{RH} ; 20 \pm 2^{\circ} \mathrm{C}$ ).

\section{Host plant exudates and honeydew collection}

To collect the honeydew, a $V$. faba plant infested with 150 aphids during $48 \mathrm{~h}$ (A. pisum or M. viciae) was placed $10 \mathrm{~cm}$ above an aluminium foil. In sterile conditions, the honeydew droplets falling on the aluminium sheet were directly collected using microcapillaries of $2 \mu \mathrm{l}$ volume. If not directly used, the samples obtained were conserved at $-20^{\circ} \mathrm{C}$.

In order to collect the host plant exudates, 15 days-old infested (150 aphids during $48 \mathrm{~h}-$ A. pisum or M. viciae -) and uninfested $V$. faba plants were used. The apical parts of plants were cut and the exudates were collected using microcapillaries of $10 \mu \mathrm{l}$ volume. The so collected exudate corresponds to a combined sample from all phloem and xylem vessels (Weibull et al. 1990). If not directly used, the samples obtained were conserved at $-20^{\circ} \mathrm{C}$.

Amino acids analyses

To analyse the honeydew free amino acid composition for the two aphid species (Modified Commission Directive 98/64/EC method for free amino acids), $50 \mu \mathrm{l}$ of fresh honeydew were used and diluted ten times in milli-Q water. Similarly, $50 \mu \mathrm{l}$ of plant exudates were used from both infested and non-infested host plants, and diluted 10 times. Deproteinization was realised by adding $500 \mu \mathrm{l}$ of a $5 \%$ solution of 5 -sulfosalicylic acid solution containing $1,000 \mathrm{nM} / \mathrm{ml}$ of norleucine (internal standard). After incubation at $4{ }^{\circ} \mathrm{C}$ during $1 \mathrm{~h}$, the samples were centrifuged during $5 \mathrm{~min}$ at $4,750 \mathrm{rpm}$. The supernatant was filtered 
$(0.2 \mu \mathrm{m})$ and finally placed in a HPLC vial. HPLC analyses were carried out on a Biochrom 20 Plus amino acids analyser (Pharmacia, Cambridge, UK, ref. 80-2111-01). Samples were injected on a cation-exchange column (Biochrom, High Performance Sodium Oxidised Feedstuff Column ref. 80-2112-18) and the amino acids were separated by elution with sodium citrate buffers of increasing $\mathrm{pH}$. Post-column derivatization was used in order to detect the amino acids at 570 and $440 \mathrm{~nm}$ (only for proline). Amino acid standard solution was prepared by mixing $4 \mathrm{ml}$ amino acid standard solution Sigma-Aldrich (Steinheim, Germany, ref. AA-S-18), $2 \mathrm{ml}$ cysteic acid $2.5 \mu \mathrm{M} / \mathrm{ml}$ sodium citrate buffer $\mathrm{pH} 2.2,2 \mathrm{ml}$ methionine sulfone $2.5 \mu \mathrm{M} / \mathrm{ml}$ sodium citrate buffer $\mathrm{pH} 2.2$ and $2 \mathrm{ml}$ norleucine $5 \mu \mathrm{M} / \mathrm{ml}$ sodium citrate buffer $\mathrm{pH} 2.2$ and by adjusting the volume to $20 \mathrm{ml}$ with sodium citrate buffer $\mathrm{pH} 2.2$ (Biochrom, ref. 80-2037-67). Amino acid standard solutions were separately injected to calibrate the analyser and quantify each amino acid. Five replications were performed for each aphid species and each exudates sample.

\section{Statistical analyses}

To compare the amino acid concentrations between the exudates of non-infested and infested plants, but also to compare the amino acid concentrations determined for the honeydews or for the plant exudates, One-way analysis of variance (ANOVA) followed by Tukey's test was used. All statistical tests were conducted by using Minitab v.15 for Windows ${ }^{\circledR}$.

\section{Results}

Amino acids analyses

The 20 amino acids, identified from all collected samples, are presented in Figs. 1 and 2.

\section{Aphid infested plant exudates versus uninfested plant exudates}

When the aphids $A$. pisum fed on the $V$. faba plant, the total amino acid concentration strongly increased (2.55 times) since the amino acids alanine, aspartic acid, histidine, isoleucine, lysine, phenylalanine, valine and the two amidated amino acids (asparagine and glutamine) significantly increased in concentration (One-way Anova analysis, Tukey's test) (Figs 1, 2). At the contrary, when considering the $M$. viciae aphids, no significant differences were noted between the amino acids from the exudates of infested and uninfested plants (One-way Anova analysis, Tukey's test)

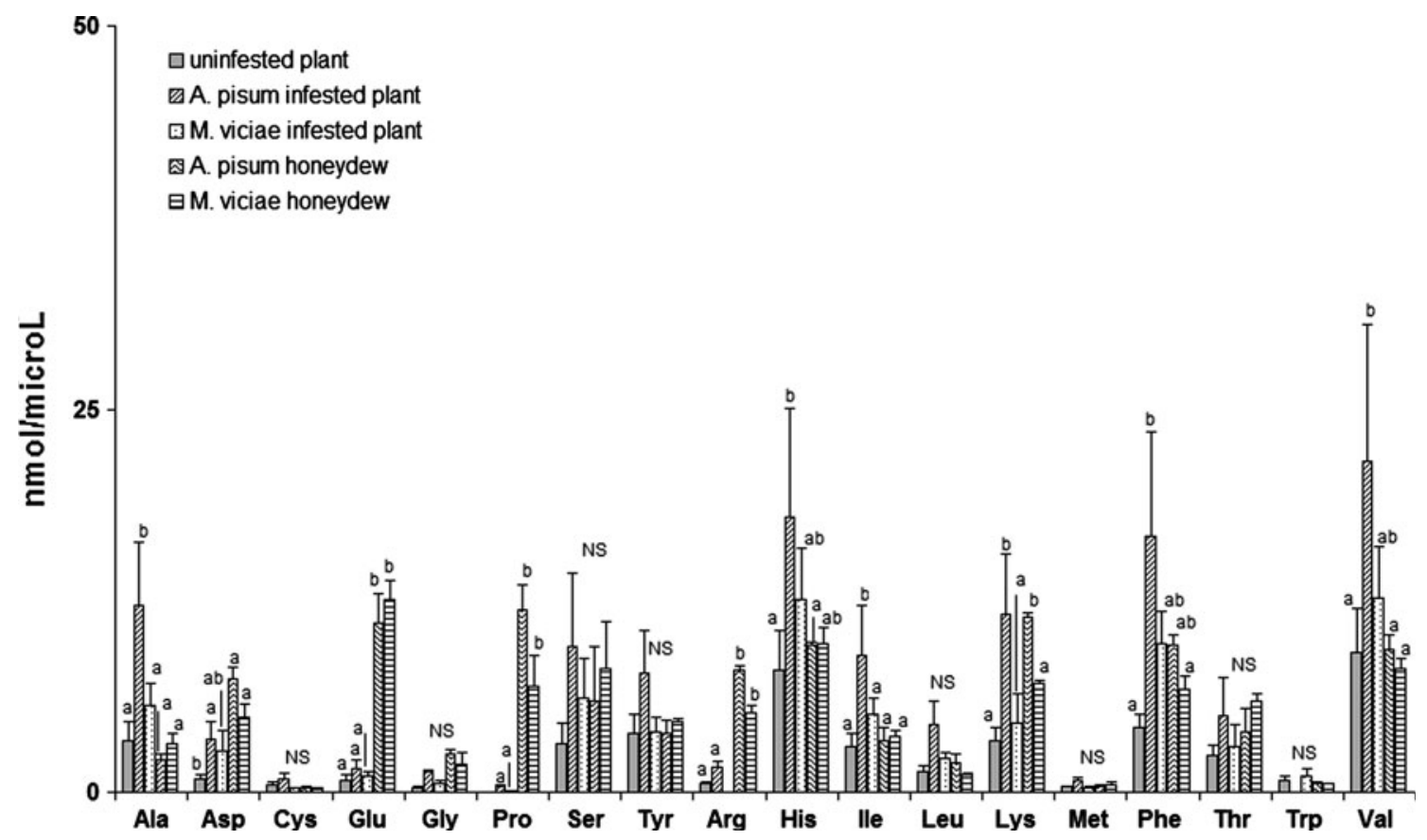

Fig. 1 Average (+SE) amino acid concentrations (Ala, Asp, Cys, Glu, Gly, Pro, Ser, Tyr, Arg, His, Ile, Leu, Lys, Met, Phe, Thr, Trp, Val) $(\mathrm{nmol} / \mu \mathrm{l})$ in plant exudates (phloem + xylem) of uninfested and infested Vicia faba plants with the aphids Acyrthosiphon pisum or Megoura viciae and amino acid concentrations $(\mathrm{nmol} / \mu \mathrm{l})$ in the
Acyrthosiphon pisum or Megoura viciae honeydews $(\mathrm{n}=5)$. For one considered amino acid, means sharing similar letters are not significantly different (Anova, Tukey's test, $P<0.05$ ). N.S. indicates "no significant difference" 


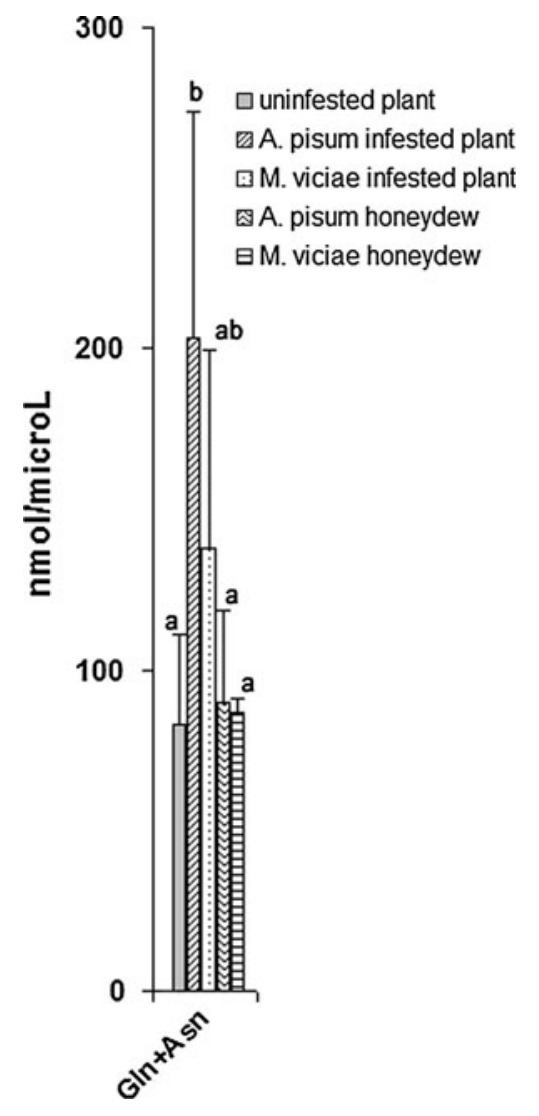

Fig. 2 Average (+SE) amino acid concentrations (Gln + Asn) $(\mathrm{nmol} / \mu \mathrm{l})$ in plant exudates (phloem + xylem) of uninfested and infested Vicia faba plants with the aphids Acyrthosiphon pisum or Megoura viciae and amino acid concentrations (nmol/ $\mu \mathrm{l})$ in the Acyrthosiphon pisum or Megoura viciae honeydews $(\mathrm{n}=5)$. Means sharing similar letters are not significantly different (Anova, Tukey's test, $P<0.05)$

and, in this case, the total amino acid concentration only increased 1.63 times.

A. pisum infested plant exudates particularly showed important modification for the amino acids glutamine (Gln) and asparagine (Asn) in comparison to the uninfested plant exudates (One-way Anova analysis, Tukey's test) (Fig. 2): glutamine and asparagine concentrations were increased 2.45 -fold and represented $61.8 \%$ of the total amino acids. The increase of Gln + Asn was less marked (1.66 times) when $M$. viciae fed on the plants, but these two amino acids also represented the most important proportion of amino acids $(65.83 \%$ of the total amino acids).

\section{Plant exudates versus aphid honeydews}

Honeydew amino acid profiles were similar for the two aphid species (One-way Anova analysis, Tukey's test) with the exception that lysine was excreted in larger amount by the aphid A. pisum (Fig. 1). The total amino acid concentrations in the honeydews were respectively $162.39 \pm 32.41$ and
$177.76 \pm 13.74 \mathrm{nmol} / \mu \mathrm{l}$ for the aphids A. pisum and $M$. viciae, smaller than the values noted for the infested plants, exudates $(329.19 \pm 151.61 \mathrm{nmol} / \mu \mathrm{l}$ for A. pisum and $209.37 \pm 82.53 \mathrm{nmol} / \mu \mathrm{l}$ for $M$. viciae). The two amidated amino acids asparagine (Asn) and glutamine (Gln) together averaged $50 \%$ of the total amino acids for the two aphid species honeydews.

When comparing the honeydew amino acid compositions to the amino acid profiles found for the infested host plants exudates (aphids intakes), the aphids A. pisum or $M$. viciae were shown to induce different kind of changes in the host plant. Compared to the amino acid concentrations determined for the infested host plant exudates, concentrations of glutamate, proline and arginine were higher for both aphid species honeydews but histidine, isoleucine, valine and the two amidated amino acids (asparagine and glutamine) were less concentrated in the A. pisum excretory product than in the infested host plants exudates (One-way Anova analysis, Tukey's test) (Figs. 1, 2). For the aphid A. pisum, it clearly appeared that the Gln + Asn concentrations strongly and significantly differed between the infested host plant exudates and the honeydew (One-way Anova analysis, Tukey's test), indicating that A. pisum took large amount of glutamine and asparagine from the host plant exudate $(203.45 \pm$ $70.18 \mathrm{nmol} / \mu \mathrm{l})$, but only excreted these two amino acids in smaller proportions $(89.53 \pm 28.69 \mathrm{nmol} / \mu \mathrm{l})($ Fig. 2). The same observation was noted for the aphid M. viciae but no significant difference was observed (One-way Anova analysis, Tukey's test): the Gln + Asn concentrations were $137.83 \pm 61.68 \mathrm{nmol} / \mu \mathrm{l}$ and $86.89 \pm 3.98 \mathrm{nmol} / \mu \mathrm{l}$, respectively for the $M$. viciae intakes and excretions (Fig. 2).

If the amino acids concentrations are compared between uninfested plant exudates and both aphid species honeydews, increases are noted for aspartate, glutamate, proline, arginine and lysine into the honeydews (One-way Anova analysis, Tukey's test) (Fig. 1).

\section{Discussion}

Amino acids are the only nitrogen source for aphids, one of the basic nutriments indispensible for growth (Mattson 1980) since insects transform amino acids into other products (Urich 1994) and use them as an energy source (Rhodes et al. 1996). Nitrogen is present in phloem sap as free amino acids of unbalanced composition with only low concentrations of most of the essential amino acids (only $20 \%$ essential amino acids) that insects cannot synthesise (Douglas 1993; Girousse et al. 1991; Sandström and Moran 1999).

For this reason, aphids ingest plant exudates at a high rate to accumulate sufficient amounts of amino acids. At 
the same occasion, aphids also intake sugars in excess, which are largely excreted into the honeydew (Douglas 2006). The role of vertically-transmitted symbionts Buchnera sp. (Douglas 2006; Shigenobu et al. 2000) that can improve aphid nutrition only by correcting the composition of ingested amino acids in phloem sap, is largely documented: symbionts essentially use glutamine and asparagine respectively converted (hydrolyzed) into glutamic acid and aspartic acid. Several different amino acids, including some essential ones (leucine, phenylalanine, valine,...), are synthesized from these four amino acids (Sasaki and Ishikawa 1995; Sasaki et al. 1993; Douglas 1998; Douglas and Prasser 1992; Wilkinson and Douglas 1995). For example, bacteria of the genus Buchnera contribute up to $90 \%$ of the essential amino acids required by the pea aphid Acyrthosiphon pisum feeding on Vicia faba, which host plant only contains $21.6 \%$ of essential amino acids according to Sandström and Pettersson (1994). In our study this percentage of essential amino acids, in the exudate of infested plants reached an average of $20 \%$.

Like other studies speculating that aphids induce changes in the composition of plant exudate in their own favour (Telang et al. 1999; Sandström et al. 2000; Sandström and Moran 2001), we confirmed that aphids alter the composition of the sap of their host plants by increasing the total concentration of amino acids. This augmentation of amino acids in the plant was observed with the aphid A. pisum, a "specialist" aphid for the host plant V. faba in contrast with the aphid M. viciae (a "generalist" aphid). Indeed, if alanine, aspartic acid, histidine, isoleucine, lysine phenylalanine, valine, glutamine and asparagine concentrations all increased when the A. pisum aphids fed on the $V$. faba plants, no significant changes were induced by the "generalist" aphid $M$. viciae. More specifically, for the pea aphid, our analyses have shown a significant increase for the amino acids glutamine and asparagine (2.45 times) while $M$. viciae did not affect the concentrations of those two amino acids. In this sense, Petersen and Sandström (2001) have shown a drastic increase (two fold) for the amino acid glutamine when the aphid Melanocallis caryaefoliae Davis fed on the pecan Carya illinoensis plant. Also, Sasaki et al. (1990) determined that glutamine was the major amino acid in the phloem sap of the bean plant on which the aphids feed. Similarly to our study, when considering the pea aphid, colonies of Aphis fabae Scopoli induced elevated levels of asparagine, aspartic acid, alanine, phenylalanine, valine and isoleucine in Vicia faba plants (Poehling 1985). In contrast to our results, this author also noted elevated levels for proline, leucine and methionine.

In addition, glutamine and asparagine were determined as the most abundant amino acids in the aphid Acyrthosiphon pisum hemolymph (Sasaki and Ishikawa 1995) but also in the phloem sap of the different host plants (Sasaki et al. 1990; Douglas 2006).

From our results, it could be assumed that the presence of aphids modifies the host plant content by enhancing the nutritional quality of the host plant exudate. Aphids are allowed to take up large amounts of glutamine and asparagine what is advantageous since other essential amino acids are synthesised from glutamine. In other studies, phloem-feeder's ability to induce changes in their host plants was demonstrated to be linked to galling (DregerJauffret and Shorthouse 1992), chlorotic lesions, necrosis (Tedders and Thompson 1981) and morphological changes (reduction in the size of roots and leaves) (Hawkins et al. 1986). These alterations are associated with changes in the phloem composition (Olmstead et al. 1997; Telang et al. 1999; Sandström 2000) and with increases in amino acid concentration in whole leaf tissue (Dorschner et al. 1987; Riedell 1989). It is also assumed that some substances in the saliva of the aphids elicit reactions in the plants inducing senescence and at the same time an increased translocation of amino acids resulting from breakdown of leaf proteins (Dorschner et al. 1987).

In this study, the amino acid quantities detected in the honeydew demonstrated that the two aphid species used glutamine and asparagine to synthesise essential amino acids. On one hand, our results showed that the concentrations of essential amino acids increased in the honeydew when compared to the exudates of uninfested plants (excepted for methionine and tryptophane): this could be explained by the direct modification of the host plant content in response to aphids on the plant, but also by the symbionts, which supply aphids with essential amino acids (Douglas 2006). Differences between the results obtained for the two aphid species certainly reflected small differences in the composition of the ingested phloem sap and/or differences in physiological capabilities of aphids or symbionts (Sandström and Moran 1999).

On the other hand, for the two aphid species, our analyses have shown that honeydew contained larger quantities of amino acids than those from the exudate of uninfested plants. Indeed, honeydews of both aphid species contained higher concentrations of aspartic acid, glutamic acid, proline and arginine than those from the exudates of infested plants.

Furthermore, when looking at the concentrations of the two amidated amino acids asparagine and glutamine, a strong difference between the exudate of infested plants and the pea aphid honeydew can be noted in contrast with the $M$. viciae honeydew. Only lower quantities of these two amino acids were excreted into the honeydew, which confirms that they were used and transformed by the symbionts after their intakes from the host plant. As showed in our study, Douglas (1993) reported that 
asparagine and glutamine were the predominant honeydew amino acids even when feeding on plants containing very low levels of these amino acids. Concentrations of glutamine and asparagine found in the honeydew corresponded to those noted for the non-infested $V$. faba plant, suggesting that aphids induce changes in the host plant when feeding to obtain large amount of these amino acids. These are later known to be metabolized into aspartate and glutamate and used by symbionts to synthesize other amino acids only present at low concentrations in the plant. Specifically, for both aphid species, proline, arginine and glutamate concentrations in the honeydew were higher than those found from the host plant exudates. This showed that symbionts have produced large amount of glutamate from the large quantity of glutamine found in the host plant in response to aphids before to be partially excreted in the honeydew.

In conclusion, this study highlights that the two aphid species (A. pisum and M. viciae) elicit different changes in the host plant exudates, showing that the "specialist" aphid A. pisum induces more important modifications in the amino acid composition of the $V$. faba host plant than the "generalist" aphid M. viciae. Concentrations of glutamine and asparagine are particularly increased when the pea aphid feeds on the plant, which is reflected in the honeydew containing large amount of glutamate produced from the large amount of glutamine absorbed. Because "specialists" only feed on one host plant, one hypothesis that could explain the different changes induced by the two aphid species is that the pea aphid is specifically adapted to the $V$. faba plant in contrast with the aphid M. viciae, a "generalist". For this reason and in an advantageous way, our study suggests that the "specialist" pea aphid is able to induce more important changes in the $V$. faba host plant in order to ingest sufficient nitrogen and adequate amount of the different amino acids.

Acknowledgments This research was funded by the Walloon Region Ministry Grant (WALEO2: SOLAPHIDRW/FUSAGX 061/6287).

\section{References}

Arakaki N, Hattori M (1998) Difference in the quality of honeydew from first instar soldier nand ordinary morph nymphs of the bamboo aphid, Pseudoregma koshunensis (Takahashi) (Homoptera: Aphididae). Appl Entomol Zool 33:357-361

Auclair JL (1963) Aphid feeding and nutrition. Ann Rev Entomol $8: 439-490$

Cloutier C (1986) Amino acid utilization in the aphid Acyrthosiphon pisum infected by the parasitoid Aphidius smithi. J Insect Physiol 32:263-267

Cull DC, van Emden HF (1977) The effect on Aphis fabae of diel changes in their food quality. Phys Ent 2:109-115

Dorschner KW, Ryan JD, Johnson RC, Eikenbary RD (1987) Modification of host nitrogen levels by the greenbug (Homoptera:
Aphididae): its role in resistance of winter wheat to aphids. Env Entomol 16:1007-1011

Douglas AE (1993) The nutritional quality of phloem sap utilized by natural aphid populations. Ecol Entomol 18:31-38

Douglas AE (1998) Nutritional interactions in insect-microbal symbioses: aphids and their symbiotic bacteria Buchnera. Ann Rev Entomol 43:17-37

Douglas AE (2006) Phloem-sap feeding by animals: problems and solutions. J Exp Bot 57(4):747-754

Douglas AE, Prasser WA (1992) Synthesis of the essential amino acid tryptophan in the pea aphid (Acyrthosiphon pisum) symbiosis. $\mathrm{J}$ Insect Physiol 38:565-568

Dreger-Jauffret F, Shorthouse JD (1992) Diversity of gall-inducing insects and their galls. In: Shorthouse JD, Rohfritsch O (eds) Biology of insect-induced galls. Oxford University Press, Oxford, pp 8-33

Faria CA, Wäckers FL, Turlings TCJ (2008) The nutritional value of aphid honeydew for non-aphid parasitoids. Basic and Appl Ecol 9(3):286-297

Febvay G, Rahbe Y, Rynkiewicz M et al (1999) Fate of dietary sucrose and neosynthesis of amino acids in the pea aphid, Acyrthosiphon pisum, reared on different diets. J Exp Biol 202:2639-2652

Fischer MK, Shingleton AW (2001) Host plant and ants influence the honeydew sugar composition of aphids. Funct Ecol 15:544-550

Fisher MK, Völkl W, Schopf R, Hoffmann KH (2002) Age-specific patterns in honeydew production and honeydew composition in the aphid Metopeurum fuscoviride: implications for ant-attendance. J Insect Physiol 48:319-326

Girousse C, Bonnemain JL, Delrot S, Bournoville R (1991) Sugar and amino acid composition of phloem sap of Medicago sativa: a comparative study of two collecting methods. Plant Physiol Biochem 29:41-48

Hawkins CDB, Whitecross MI, Aston MJ (1986) Interactions between aphid infestation and plant growth and uptake of nitrogen and phosphorus by three leguminous host plants. Can J Bot 64:2362-2367

Hendrix DL, Wie Y, Leggett JE (1992) Homopteran honeydew sugar composition is determined by both the insect and plant species. Comp Biochem Physiol A 101B:23-72

Hogervorst P, Wäckers F, Romeis J (2007) Effects of honeydew sugar composition on the longevity of Aphidius ervi. Entomol Exp Appl 122:223-232

Karley AJ, Douglas AE, Parker WE (2002) Amino acid composition and nutritional quality of potato leaf phloem sap for Aphids. J Exp Biol 205:3009-3018

Malcolm SB (1990) Chemical defence in chewing and sucking insect herbivores: plant-derived cardenolides in the monarch butterfly and oleander aphid. Chemoecology 1:12-21

Mattson WJ (1980) Herbivory in relation to plant nitrogen content. Annu Rev Ecol Syst 11:119-161

Mittler TE (1958) Studies on the feeding and nutrition of Tuberolachnus salignus II the nitrogen and sugar composition of ingested phloem sap and excreted honeydew. J Exp Biol 35:74-84

Olmstead KL, Denno RF, Morton TC, Romeo JT (1997) Influence of Prokelisia plant hoppers on amino acid composition and growth of Spartina alterniflora. J Chem Ecol 23:303-321

Petersen MK, Sandström JP (2001) Outcome of indirect competition between two aphid species mediated by responses in their common host plant. Funct Ecol 15:525-534

Poehling HM (1985) Einfluss von Aphis fabae Scop (Homoptera, Aphididae) auf den Protein und Aminosäurestoffwechsel von Vicia faba Mitteilungen der Deutschen Gesellschaft für Allgemeine und Angewandte. Entomol 4:366-369 
Prosser WA, Douglas AE (1991) The aposymbiotic aphid: an analysis of chlortetracycline-treated pea aphid, Acyrthosiphon pisum. J Insect Physiol 37:713-719

Rhodes JD, Croghan PC, Dixon AFG (1996) Uptake, excretion and respiration of sucrose and amino acids by the pea aphid Acyrthosiphon pisum. J Exp Biol 199:1269-1276

Riedell WE (1989) Effect of Russian wheat aphid infestation on barley plant response to drought stress. Physiol Plant 77:587-592

Saheed SA, Botha CEJ, Liu L, Jonsson L (2007) Comparison of structural damage caused by Russian wheat aphid (Diuraphis noxia) and Bird cherryoat aphid (Rhopalosiphum padi) in a susceptible barley cultivar, Hordeum vulgare cv. Clipper. Physiol Plant 129:429-435

Sandström J, Moran N (1999) How nutritionally imbalanced is phloem sap for aphids? Entomol Exp Appl 91:203-210

Sandström J, Moran N (2001) Amino acid budgets in three aphid species using the same host plant. Physiol Entomol 26(3): 202-211

Sandström J, Pettersson J (1994) Amino acid composition of phloem sap and the relation to intraspecific variation in pea aphid (Acyrthosiphon pisum) performance. J Insect Physiol 40: 947-955

Sandström J, Telang A, Moran NA (2000) Nutritional enhancement of host plants by aphids-a comparison of three aphid species on grasses. J Insect Physiol 46:33-40

Sasaki T, Ishikawa H (1995) Production of essential amino acids from glutamate by mycetocyte symbionts of the pea aphid, Acyrthosiphon pisum. J Insect Physiol 41:41-46

Sasaki T, Aoki T, Hayashi H, Ishikawa H (1990) Amino acid composition of the honeydew of symbiotic and aposymbiotic pea aphids Acyrthosiphon pisum. J Insect Physiol 36:35-40

Sasaki T, Fukuchi N, Ishikawa H (1993) Amino acid flow through aphid and its symbiont: studies with 15 N-labeled glutamine. Zool Sci 10:787-791

Shigenobu S, Watanabe H, Hattori M et al (2000) Genome sequence of the endocellular bacterial symbiont of aphids Buchnera sp. APS. Nature 407:81-86

Smith KM (1926) A comparative study of the feeding methods of certain Hemiptera and of the resulting effects upon the plant tissue, with special reference to the potato plant. Ann Appl Biol 13:109-139

Smith DC, Douglas AE (1987) The biology of symbiosis. Edward Arnold London

Spiller NJ, Koenders L, Tjallingii WF (1990) Xylem ingestion by aphid-a strategy for maintaining water balance. Entomol Exp Appl 55:101-104
Tedders WL, Thompson JM (1981) Histological investigation of stylet penetration and feeding damage to pecan foliage by three aphids (Hemiptera (Homoptera): Aphididae). In: Johnson D (ed) Tree fruit and nut pest management in the Southeastern United States. Entomological Society of America, Lanham, pp 69-83

Telang A, Sandström J, Dyreson E, Moran NA (1999) Feeding damage by Diuraphis noxia results in a nutritionally enhanced phloem diet. Entomol Exp Appl 91:403-412

Tjallingii WF (1994) Regulation of phloem sap feeding by aphids. In: Chapman GF, De Beor G (eds) Regulatory mechanisms in insect feeding. Chapman and Hall, New York, pp 190-209

Urich K (1994) Comparative animal biochemistry. Springer-Verlag, Berlin

Wäckers FL (2000) Do oligosaccharides reduce the suitability of honeydew for predators and parasitoids? A further facet to the function of insect-synthesized honeydew sugars. Oikos 90: 197-201

Wäckers FL (2005) Suitability of (extra-) floral nectar, pollen, and honeydew as insect food sources. In: Wäckers FL, van Rijn PCJ, Bruin J (eds) Plant-provided food for carnivorous insects: a protective mutualism and its applications. Cambridge University Press, Cambridge, pp 17-74

Way MJ (1963) Mutualism between ants and honeydew-producing Homoptera. Annu Rev Entomol 8:307-344

Weibull J, Ronquist F, Brishammar S (1990) Free amino acid composition of leaf exudates and phloem sap. Plant Physiol 92:222-226

Wilkinson TL, Douglas AE (1995) Why pea aphids (Acyrthosiphon pisum) lacking symbiotic bacteria have elevated levels of the amino acid glutamine. J Insect Physiol 41(11):921-927

Wilkinson TL, Ashford DA, Pritchard J, Douglas AE (1997) Honeydew sugars and osmoregulation in the pea aphid Acyrthosiphon pisum. J Exp Biol 200:2137-2143

Woodring J, Wiedemann R, Fischer MK et al (2004) Honeydew amino acids in relation to sugars and their role in the establishment of ant-attendance hierarchy in eight species of aphids feeding on tansy (Tanacetum vulgare). Physiol Entomol 29:311-319

Yao I, Akimoto SI (2001) Ant attendance changes the sugar composition of the honeydew of the drepanosiphid aphid Tuberculatus quercicola. Oecologia 128:36-43

Yao I, Akimoto SI (2002) Flexibility in the composition and concentration of amino acids in honeydew of the drepanosiphid aphid Tuberculatus quercicola. Ecol Entomol 27:745-752 